\title{
Corela
}

Cognition, représentation, langage

6-2 | 2008

Vol. $6, n^{\circ} 2$

\section{Le langage : une empreinte cognitive}

\section{Nicolas Bourguignon}

\section{OpenEdition}

\section{Journals}

Édition électronique

URL : http://journals.openedition.org/corela/317

DOI : $10.4000 /$ corela.317

ISSN : $1638-573 \mathrm{X}$

\section{Éditeur}

Cercle linguistique du Centre et de I'Ouest - CerLICO

\section{Référence électronique}

Nicolas Bourguignon, « Le langage : une empreinte cognitive », Corela [En ligne], 6-2 | 2008, mis en ligne le 15 décembre 2008, consulté le 10 décembre 2020. URL : http://journals.openedition.org/ corela/317 ; DOI : https://doi.org/10.4000/corela.317

Ce document a été généré automatiquement le 10 décembre 2020.

\section{(c) (i) (2)(2)}

Corela - cognition, représentation, langage est mis à disposition selon les termes de la licence Creative Commons Attribution - Pas d'Utilisation Commerciale - Partage dans les Mêmes Conditions 4.0 International. 


\title{
Le langage : une empreinte cognitive
}

\author{
Nicolas Bourguignon
}

\section{Introduction}

1 Dans le cadre de son Programme d'étude de la cognition sociale(2003) ${ }^{1}$, le linguiste Ray Jackendoff perçoit dans le concept d'organisation sociale le trait caractéristique d'une majorité d'espèces animales. Ce phénomène, quoique différent d'espèce en espèce, demeure une réalité pour l'essentiel assez stable au cœur de celles-ci. Mais dans le vaste panorama des populations terrestres, l'homme moderne et sa nature pour le moins singulière font figure d'exception. Au contraire des chimpanzés, nos cousins les plus proches dans la superfamille des hominoïdes, pour qui les habitudes sociales et culturelles ne se distinguent d'un groupe à l'autre qu'à très peu d'égards, les peuples humains connaissent des sous-ensembles très divers d'usages partagés et transmis. Cette variété culturelle significative figure traditionnellement parmi les attributs nécessaires évoqués par les ethnologues en vue de différencier l'homme de l'animal (Tattersall, 1998). Le défi majeur auquel tout individu se trouvera confronté au fil de son existence sera d'amasser un ensemble large et hétérogène d'indices culturels pour à terme en acquérir une connaissance productive et appropriée.

2 Que les linguistes prêtent l'oreille aux enseignements des ethnologues apparait aujourd'hui bien moins surprenant qu'évident. A la lumière des progrès réalisés dans l'observation des phénomènes humains, tant les uns que les autres ne sauraient éviter un parallèle fondamental entre leurs disciplines. Le problème de l'acquisition de la compétence culturelle et sociale, suggère Jackendoff, ne va pas sans évoquer celui de l'acquisition du langage exposé en 1965 par Noam Chomsky dans son ouvrage fondateur Aspects of the Theory of Syntax. Dans les deux cas de figure, l'enfant est amené, dès les premières années, à acquérir des structures éminemment complexes, sans contours nets ni définis par avance. Cette tâche s'avère d'autant plus ardue que langage et culture constituent tous deux des phénomènes d'une grande variabilité. Ainsi, si la culture ne cesse chaque jour de s'enrichir et de se modifier, les signes linguistiques et les systèmes 
dont ils font partie n'échappent pas non plus au changement. C'est probablement cette intuition qui guida la plume de Wittgenstein (1961, p. 24) lorsqu'il écrivit qu'« il est d'innombrables et diverses sortes d'utilisation de tout ce que nous nommons 'signes', 'mots', 'phrases'. Et cette diversité, cette multiplicité n'est rien de stable, ni de donné une fois pour toutes.»

3 La beauté du rapprochement entre linguistique et ethnologie est manifeste, et se ressent principalement dans l'effort de comprendre et décrire deux aspects remarquables de la nature humaine. Le consensus apparaît d'ailleurs plus clairement si l'on accepte que le langage figure parmi les vecteurs par excellence de transmission culturelle. Ignorer l'un au profit de l'autre serait une lacune indéniable dans nos connaissances. Toutefois, la recherche des nombreux points de contact entre la linguistique et toute autre science potentiellement susceptible de l'enrichir ne devrait pas nous amener à évincer la frontière entre ce les rassemble et ce qui les distingue. En d'autres termes, il serait tout aussi regrettable que la proximité séduisante entre langage et culture nous empêche d'étudier l'un sans se départir de l'autre. Et bien que cette prise de distance soit un mal nécessaire pour les uns, elle apparaît bien moins évidente pour les autres.

4 L'acquisition d'un langage humain est-elle le résultat d'un apprentissage inductif, indistinct des autres produits de l'esprit, et simplement dû à notre « curiosité naturelle ", à notre " prédisposition à développer des idées nouvelles » et à « interroger la Nature au travers d'expériences concrètes » (Sampson, 2005, p.2) ${ }^{2}$ ? Au cœur de cette problématique réside un débat connu de longue date, opposant la conception empiriste des langues comme phénomènes abstraits, forgés dans le creuset d'une communauté culturelle donnée, et celle du langage comme produit cognitif interne, propre à la biologie des locuteurs.

Observer les évènements marquant l'histoire des sciences depuis le XVIIe siècle nous permet aujourd'hui de poser un premier constat: Depuis l'énonciation des prolégomènes empiristes de Locke et de leurs antithèses nativistes contemporaines (Descartes constitue ici un représentant de choix); malgré l'échec cuisant des tentatives béhavioristes d' «appliquer les principes du conditionnement opérant sur les capacités langagières humaines " (Fisette, Poirier, 2000) face aux méthodes récentes et novatrices du programme générativiste, le débat nature vs nurture demeure à ce jour sans issue satisfaisante. Le rapport du langage au monde est si présent qu'il demeure difficile d'envisager son acquisition sans admettre la moindre interaction entre le locuteur et son milieu culturel et social. Bien qu'il soit impensable de mettre un terme strict et définitif à cette problématique aujourd'hui, je souhaiterais décrire ici quelques idées développées très récemment, qui tout en préservant l'idée du langage comme part de notre patrimoine génétique, englobent aussi la dimension éthologique de celui-ci d'une manière qui me semble élégante - peut-être même conciliante, en particulier lorsqu'il s'agit de savoir «comment» le locuteur s'appuie sur le monde pour développer sa compétence linguistique.

6 Dans cette optique, il m'a été indispensable d'illustrer les présents arguments en adoptant un point de vue vaste et pluridisciplinaire, de manière à ce que chacun puisse identifier sa place dans l'étude du langage. Dans un premier temps, je soulèverai la problématique relativiste esquissée dans la célèbre hypothèse Sapir-Whorf, qui bien qu'ayant suscité nombre de controverses, colore encore aujourd'hui la perception de nombreux faits linguistiques observés à travers le monde (Gordon, 2004; Özgen, 2004). A l'instar de nombreuses critiques, le présent texte en privilégiera notamment une lecture inverse, et 
proposera que la diversité linguistique n'est somme toute que le reflet de la richesse conceptuelle avec laquelle l'homme est capable d'appréhender son milieu. En m'appuyant sur de récents arguments issus de faits aujourd'hui reconnus dans les sciences cognitives, je préciserai pour ma part que cette même richesse pourrait dans les faits constituer la base cognitive sur laquelle les langues se seraient développées. A partir de là, je tenterai de circonscrire le rôle du langage dans la perspective plus large de ce que nous nommons communément la communication. Cette démarche se justifie par les nombreuses suggestions théoriques et empiriques proposées dans le cadre des sciences cognitives, de la linguistique et de la psychologie du développement, qui nous indiquent notamment que certaines pathologies étudiées de longue date mais toujours empreintes de mystère suscitent l'intérêt grandissant des psycholinguistes dans leur exploration des propriétés nécessaires à la capacité de communication, que ces dernières soient d'ordre culturel, social ou cognitif. Enfin, dans la mesure où ma réflexion ne saurait résolument trouver de frontière entre les disciplines, j'encadrerai la présente discussion dans l'esprit des récentes questions soulevées par les sciences cognitives dans l'histoire du langage et de l'humanité.

\section{Point de départ : le concept avant la lettre}

7 Bien qu'il ne soit nullement dans mes objectifs de développer ici une nouvelle critique détaillée du relativisme linguistique d'Edward Sapir (1949) et Benjamin Lee Whorf (1956), je souhaiterais prendre comme point de départ à ma réflexion l'observation fondamentale qui amena en premier lieu E. Sapir à postuler l'existence d'une réalité " dans une grande mesure inconsciemment construite à partir des habitudes langagières du groupe (Sapir, 1951, p. 1962) ». L'investigation des langues amérindiennes au début du XXe siècle suggérait à Sapir que les différences structurelles et lexicales des langues du monde, dans leur façon de dépeindre la réalité, n'avaient d'autre effet que de " déterminer la nature et le contenu de la pensée » (in Gordon, 2004). Poussée au plus loin dans le déterminisme linguistique de B. L. Whorf, l'idée de Sapir tendait d'une certaine façon à s'inscrire dans la conception saussurienne des langues comme autant de systèmes de découpage arbitraire de la réalité, ce à quoi personne ne devrait en principe s'opposer dans la mesure où les mots que nous employons ne sont au final que des estampes, des empreintes plus ou moins représentatives d'une réalité elle-même profondément variable, non seulement de groupe en groupe, non seulement au niveau physique, mais aussi chez chacun d'entre nous (comme l'observe Michel Seymour ${ }^{3}$, que deux personnes partagent une même langue n'implique aucune exclusivité de connaissance). Le problème fondamental que révèle l'hypothèse Sapir-Whorf est de proposer une influence directe et non réciproque de la forme linguistique sur la substance de l'esprit, sans toutefois envisager que cette dernière ait à l'inverse été à l'origine de la première. En dépit des nombreuses expériences ayant tenté de confirmer ou d'infirmer cette idée de base (l'étude de Gordon 2004 auprès des Pirahã en est d'ailleurs une récente illustration), il demeure qu'un lien direct de causalité entre langage et perception semble bien loin d'être une réalité.

8 Sur le point de la relation entre langage et conceptualisation, la tradition générativiste (Pinker, 1995; Chomsky, 1957, 1964) n'a eu de cesse de privilégier une coupure nette entre les deux sphères, arguant qu'une étude indissociée de la forme par rapport à la sémantique ne serait à même de refléter fidèlement la compétence linguistique universelle 
des individus, à commencer par leur aptitude à maîtriser instinctivement la grammaire de leur langue pour interpréter et produire un nombre potentiellement infini de phrases inédites dans les premières années de vie. Cette intuition se fonde davantage que cette prédisposition apparaitt homogène " en dépit d'une exposition très partielle et imparfaite de l'enfant aux variations linguistiques possibles de sa langue », voire « des disparités de l'environnement culturel et affectif »(Mercier, 2007). C'est à partir de ces observations qu'une étude cognitive du langage fut suggérée. Dans l'ancrage des premières suggestions saussuriennes, la linguistique générative cherche depuis son avènement à fournir une explication "mentaliste» du langage (Culicover, Jackendoff, 2005), à proposer une description adéquate des règles formelles internes à notre « grammaire mentale ».

Il ne fait aujourd'hui aucun doute qu'une étude abstraite des langues impliquant une distanciation par rapport aux concepts sémantiques qu'elles expriment permit une avancée considérable dans l'observation de données linguistiques issues d'une grande variétés de langues, si bien que certains pourraient se satisfaire de l'outil d'investigation développé par la grammaire générative sans pour autant adhérer intégralement aux visions nativistes ou universalistes qu'elle présuppose. Cependant, plusieurs questions s'imposent d'emblée, lesquelles ne pourraient trouver de réponse satisfaisante si l'on omettait le rapport indubitable que le langage entretient avec le monde. Parler une langue implique un remarquable savoir-faire, permettant de faire naître des idées nouvelles et complexes dans l'esprit de nos interlocuteurs avec une extrême précision (Pinker, 1994). Cette faculté semble d'autant plus impressionnante que ces idées constituent des entités mentales internes, abstraites, lesquelles naissent, "vivent et meurent à l'intérieur de nos esprits, sans jamais en sortir» (Sperber-Wilson 2002; Sperber-Wilson, 1981). D'autre part, si ces idées peuvent à terme s'échanger au travers d'énoncés linguistiques, cela ne signifie pas qu'elles sont présentes dans notre esprit sous une forme linguistique préétablie, ce qui suppose de toute évidence un mécanisme "pivot» entre les niveaux de représentation. Peu importe l'autonomie du langage par rapport au concept, celle-ci ne représente en aucun cas une condition suffisante à la communication. A un moment donné, il nous est indispensable d'envisager ce que Jackendoff (2007) nomme une "architecture parallèle » entre ces deux réalités. Deux points de vue particuliers permettent en outre d'envisager que de ces deux systèmes, l'un serve d'assise à l'émergence de l'autre : au niveau développemental, tout d'abord, il est souvent acquis que la sphère conceptuelle prime sur la maitrise de toute catégorie grammaticale et des règles syntaxiques auxquelles elle est soumise (de Boysson-Bardies, 1996, chap. 3). Du point de vue de l'évolution, la question se pose par ailleurs de savoir comment un système linguistique si complexe aurait pu apparaitre avant toute perception symbolique; une problématique rarement soulevée par les linguistes, mais tout à fait pertinente au regard des anthropologues (Tattersall, 2007 ; Dubreuil, 2008), pour qui les représentations artistiques des premières populations évoluées d'homo sapiens, "étaient au centre de leur façon de percevoir leur environnement et de s'expliquer le monde, ainsi que - sans doute - leur position au sein de celui-ci» (Tattersall, 1998). Les faits deviennent encore plus informatifs lorsque l'on sait que ces représentations requéraient naturellement une sophistication des techniques, dont le développement aurait pu se produire « de concert avec la diversification linguistique et l'apparition des premiers dialectes locaux" (Ibid). Comme l'indique la remarque de Jackendoff (2003), il serait difficilement acceptable pour un linguiste de rester indifférent à ce genre de scénario. 
"If you could imagine hominids evolving a syntactic capacity without any

connections to sound and meaning, what good would it do?» que la pauvreté du stimulus linguistique ne permet pas à l'individu de bâtir sa faculté du langage, la richesse d'informations non linguistiques fournies par l'environnement du locuteur, de même que sa prédisposition à catégoriser symboliquement le monde, voire à associer les mots de sa langue aux concepts qu'ils encodent sont autant de préalables à toute compétence linguistique. Cette dernière assomption, si elle se confirme, justifierait fort bien que des composantes mentales souvent associées à la sphère paralinguistique aient elles aussi un rôle à jouer dans l'ontologie des grammaires naturelles.

\section{La communication : une structure à plusieurs niveaux}

11 Le concept d'une faculté communicative plus générale et complexe que celle exclusivement impliquée dans production d'énoncés linguistiques n'est pas chose nouvelle. En vérité, cet «organe mental » (Lightfoot, 1987) que constitue la grammaire d'une langue naturelle n'aurait somme toute que peu d'utilité si celui-ci n'interagissait avec d'autres mécanismes spécialisés dans l'exécution de tâches cognitives sous-jacentes. Par ailleurs, la distinction entre langue est pensée est pour beaucoup devenue une pure évidence :

"Linguistics has shown that human languages are too ambiguous and schematic to use as a medium of internal computation; when people think about "spring," surely they are not confused as to whether they are thinking about a season or something that goes "booing" - and if one word can correspond to two thoughts, thoughts can't be words. (Pinker, 1995, p. 136). "

12 Ces observations ont naturellement incité de nombreux scientifiques à se pencher sur l'implication des mécanismes d'extraction du sens impliqué dans un message linguistique. Sur la base des travaux effectués dans le cadre de la linguistique générative, le philosophe américain Fodor (1984) propose d'associer les idées à des entités mentales internes, non linguistiques, constituant notre "langage résidant». Ces systèmes de représentation se transmettent au moyen d'un code, que le locuteur utilisera pour traduire les agencements de mots en combinaisons d'idées, et inversement, tout au long de l'échange linguistique. Cette conception semble se confirmer par les nombreuses études des processus de compréhension proposées par Schank (1977) dans le courant des années 1970, et résumées par Auroux, Deschamps et Kouloughli (2004):

« Dans ses travaux, Schank constate que les humains sont très rarement capables de se souvenir avec exactitude de la forme linguistique dans laquelle on leur a communiqué une information. Ce dont ils se souviennent, c'est du «contenu » de l'information, ce qui suggère que les humains traduisent spontanément l'information linguistique en ce que Schank appelle des représentations conceptuelles, lesquelles sont indépendantes des structures syntaxiques et sans doute même du lexique particulier d'une langue naturelle.»

13 A ce stade, rien ne nous explique la façon dont ces systèmes de représentations seront à terme intégrés dans des combinaisons linguistiques, ni extraites de celles-ci. Ainsi, comme l'indiquent Sperber et Wilson (1981), l'interprétation d'un message ne s'arrête pas au simple décodage de l'information linguistique en représentations conceptuelles. Une fois le message transmis et perçu, il incombe à l'interlocuteur de retirer l'information 
effectivement impliquée dans le message (Pinker, 1994). Et ce décodage sous-jacent n'est possible que par l'intervention de processus inférentiels "différents en nature des processus de décodage linguistique » (Sperber-Wilson, 1981).

14 Nous voici déjà en présence de deux modules spécifiques mais concurrents : l'un destiné à l'interprétation linguistique (le modèle du code), l'autre à l'interprétation inférentielle. Il apparait évident que cette dernière interprétation ne sera permise que si le locuteur s'appuie sur un ensemble d'indices extralinguistiques comme les conventions sociales, les coutumes, les objets et les faits de l'environnement éventuellement désignés par la phrase et ses constituants, mais ce constat n'enlève en rien la nécessité de comprendre la vraie nature des phénomènes permettant au locuteur de découvrir les relations entre ces indices et leurs équivalents linguistiques. Une approche, proposée par Slobin (1986), serait d'envisager l'existence de Principes Opératoires de regroupement :

«Although we have no direct evidence for the basic strategies for grouping information in linguistic storage, some Operating Principles can be proposed as at least minimal prerequisites. In a broad view, salient dimensions for system building include phonological similarity, referential meaning, pragmatic function, and formal patterning (in Peters, 1986, p. 309).»

15 Aux modules codique et inférentiel décrits plus haut, je souhaiterais en ajouter un troisième, lequel me sera utile pour mes arguments ultérieurs. J'illustrerai ce mécanisme au moyen de deux courts passages, l'un tiré du célèbre article Meaning, rédigé en 1957 par le philosophe et fondateur de la pragmatique Paul Grice, l'autre de l'article Chimpanzee Theory of Mind: A Proposal from the Armchair, de Sam Scott, professeur au département de sciences cognitives à l'université d'Ottawa (2001). Dans le premier article, Grice se livre à une description plutôt schématique mais néanmoins élégante du processus de signification lors d'un acte de communication entre une personne $A$ et ses interlocuteurs :

"A meant something by $x$ ' is (roughly) equivalent to 'A intended the utterance of $x$ to produce some effect in an audience by means of the recognition of this intention.»

Dans le second passage, l'auteur relate les observations faites par l'anthropologue Gómez (1991) sur le développement d'une jeune femelle gorille, et notamment sur sa capacité à transmettre certains de ses désirs aux expérimentateurs.

" The gorilla was tested repeatedly in a zoo nursery between the ages of 6 to 30 months. The task was to open a door by undoing a latch placed high up beyond her reach. The only other objects in the room were a box (which if the gorilla stood on it would enable her to reach the latch) and an adult human experimenter. By the end of the first year, the gorilla had figured out that she could move the box over to the door and climb on it to reach the latch. Very soon after this cognitive breakthrough, she began to use the human as a box, pushing and pulling roughly until he was near the door, and then climbing him to reach the latch.

Finally, at about 18 months, the gorilla began communicating with the human. She would lead him gently by the hand over to the door, where she would extend his hand towards the latch and repeatedly look from his eyes to the latch and back again as though asking him to open the door. »

34 ans séparent l'article de Grice des observations de Gómez, et pourtant, tant Grice que Gómez rendent compte d'une même faculté, celle d'associer les personnes de notre environnement à des « systèmes intentionnels » (Scott, 2001, p. 3), dont les états mentaux (croyances, désirs, joie, peur, etc.) ne nous seraient aucunement accessibles en l'absence de divers indicateurs d'intention communicative. Cette faculté, proposée pour la première fois par les anthropologues Premack et Woodruff (1978) sous le vocable de 
« théorie de l'esprit », a rapidement suscité un vif intérêt dans le cadre des recherches sur la communication humaine.

18 C'est en particulier le cas des nombreuses études sur l'autisme conduites par les psychologues britanniques Simon Baron-Cohen et Heather van der Lely (1996). Les troubles d'interaction sociale et le déficit communicatif caractéristiques de l'autisme ont notamment amené Baron-Cohen, Leslie et Frith (1985) à poser l'hypothèse que ces populations étaient en réalité incapables d'interpréter les états mentaux d'autrui (le mindblindness selon Baron-Cohen), ce qui expliquerait plusieurs aspects inhabituels de leur comportement verbal. De manière générale, Fine et coll. (1994) soulignent une lacune criante quant à l'usage du langage en contexte dans la population autiste. Non seulement leur langage littéral et écholalique rend-il difficile, voire impossible, toute conversation, mais ce handicap se ressent aussi dans plusieurs aspects essentiellement pragmatiques de leur compétence linguistique. Pour ne citer que quelques exemples, Baltaxe $(1977,1984)$ et Fay (1979) évoquent entre autres un usage incorrect des pronoms personnels ; Peppé, et coll. (2006) détectent une incapacité à percevoir certaines altérations de la prosodie, que celle-ci soit affective (affect, attitudes, émotions, etc.), ou grammaticale, (incapacité de percevoir les variations d'intonation entre une phrase affirmative [Tu viens avec.] et interrogative [Tu viens avec ?], difficulté à segmenter le continuum sonore en unités plus restreintes, intonation, accent contrastif). Cependant, dans une étude sur les processus syntaxiques de transformations chez cinq enfants autistes, Pierce et Bartolucci (1977) affirment que leurs sujets maitrisaient en grande partie la syntaxe de leur langue. Sur la base d'une comparaison entre enfants autistes et enfants dysphasiques sur leur aptitude à identifier la violation des maximes de conversation de Grice, Surian et coll. (1996) ont pour leur part conclu que, bien qu'accusant un important déficit dans le développement de la pragmatique, les autistes de haut niveau ne présentaient, à tout le moins, qu'un léger retard morphologique et syntaxique. Pour ma part, de telles conclusions me semblent pour le moins hâtives. Et pour cause, je soulignerai d'emblée que si les pronoms personnels posent effectivement un problème pragmatique aux enfants autistes en amont, il est possible que cela ait un impact en aval sur les Principes A ou B de la théorie Gouvernement-Liage de Chomsky qui régissent l'usage syntaxique des pronoms et des anaphores (Chomsky, 1981, 1995) ; une question jusqu'alors non testée empiriquement, peut-être difficile, mais tout à fait raisonnable.

19 Observer le langage sous cet angle implique évidemment que l'on adopte un point de vue plus large qu'auparavant, en ce sens qu'il s'agit là d'intégrer certaines notions parallèles au langage proprement dit. Il est tout à fait clair que la capacité inférentielle et la théorie de l'esprit sont aussi mises en œuvre à d'autres fins que celle de communiquer - la preuve étant que celles-ci semblent se retrouver (même sous une forme rudimentaire) chez d'autres espèces animales. Sperber et Wilson (2002) proposent pour leur part que la théorie de l'esprit fait partie intégrante de notre faculté pragmatique (notamment inférentielle), mais je préciserai pour ma part qu'une analyse plus poussée de la théorie de l'esprit lui attribuerait un rôle supérieur à la reconnaissance d'une intention communicative. Il n'est pas impossible même que celles-ci participent de façon inhérente à la sphère spécifique du langage. A ce titre, la récente approche proposée par Chomsky, Hauser et Fitch (2002) semble nous ouvrir la voie :

«Faculty of language - broad sense (FLB)includes an internal computationalsystem (FLN, below) combined withat least two other organism-internal systems, which we call "sensory-motor" and "conceptual-intentional." [...]Faculty of language-narrow sense (FLN) is the abstract linguistic computationalsystem alone, independent of the 
othersystems with which it interacts and interfaces.FLN is a component of FLB, and themechanisms underlying it are some subset ofthose underlying FLB. "(Chomsky, Hauser et Fitch, 2002) joue chacun des «modules » cognitifs dans notre compétence communicative, plus nous acquerrons une idée plus claire de ce que représente le langage au sens strict. Au niveau technique, une démarche devenue sans aucun doute légitime consiste - comme le propose Robert Van Valin (2005) - à envisager une projection pragmatique en connexion avec les projections syntaxiques ou interprétatives (logiques) du langage, qui agirait par elle-même sur la structure de l'information transmise dans le message, de telle sorte qu'on ne pourrait l'exclure de la grammaire. S'il en est ainsi au niveau théorique, une compétence pragmatique déficiente n'aurait-elle pas, à quelque égard, un impact sur certains aspects de leur compétence morphosyntaxique semblables à l'usage des pronoms et anaphores soulevé plus haut?

21 A nouveau, si les suppositions que j'ai proposées relèvent du possible, découvrir comment l'individu systématise son environnement pourrait nous fournir le cadre de travail adéquat pour l'étude des Universaux Sémantiques ${ }^{4}$. Pour beaucoup, ces derniers sont un fait incontournable. Chomsky (1968) raisonne dans un esprit similaire lorsqu'il suggère que «l'interprétation sémantique d'une phrase est déterminée par des principes universels, indépendants du langage » et " reliés grammaticalement aux concepts compris dans les énoncés ${ }^{5}$ ", mais comme il le souligne, cette hypothèse est, jusqu'alors, restée majoritairement «tacite» (Malgré les propositions intéressantes de Lenneberg, 1967). Si ceci est certes vrai pour la linguistique moderne, il n'en va certainement pas de même pour la psychologie cognitive et évolutionniste, qui pour sa part s'attèle à découvrir les régularités du comportement humain. En ce sens, il ne pourrait être question d'aborder la problématique des universaux sémantiques et de leur lien avec notre compétence grammaticale sans une connaissance approfondie des «fondations psychologiques de la culture » (Tooby, Cosmides, 1992).

Comment mettre notre démarche en pratique ? Étonnamment, la littérature sur le sujet même parmi les défenseurs du générativisme - regorge d'informations plus qu'intéressantes. Ainsi, dans ses travaux d'acquisition de la syntaxe chez les enfants normaux, Pinker (1987) propose par exemple qu'un enfant infère les catégories grammaticales de sa langue (et fonde dès lors sa compétence syntaxique) sur la base d'un premier découpage de son environnement. Pour faire une histoire courte, il suffirait à l'enfant d'entendre un énoncé $\mathrm{E}$ (par exemple Marie frappe la balle) dans une situation S, lors de laquelle un AGENT frappe un THÈME, pour identifier par heuristique le rôle joué par chacun des composants, de même que l'ÉVÉNEMENT en question : celui consistant à frapper une balle. Cette explication a ses limites, comme toutes celles qui tendent à expliquer l'acquisition du langage, mais est d'une rare élégance. La part innée tient autant dans l'analyse d'une situation externe, douée d'une grande richesse d'informations visuelles et auditives, que dans la capacité à associer ces "priorités épistémologiques " (Chomsky, 1981 ; développé dans Pesetsky, 1982) à des relations structurales et - à terme - syntaxiques. Il conviendrait bien entendu d'approfondir le sujet d'un point de vue strictement théorique, notamment en regard des différences interlinguistiques (pour une discussion sur le sujet, voir Baker 1997), mais il parait difficile d'envisager une autre façon d'acquérir le sens (et donc une catégorie grammaticale potentielle) d'un élément aussi abstrait qu'un verbe. Cette piste d'exploration pourrait être tout aussi valable dans 
l'étude du langage autiste : si des événements aussi tangibles que ceux qui composent le milieu de l'enfant lui sont effectivement utiles pour asseoir sa compétence grammaticale, il devrait en être autant pour la compréhension des prédicats psychologiques (avoir peur, être triste, douter voire le sens métaphorique d'être frappé par une horrible nouvelle), ce qui requerrait alors (à tout le moins au niveau conceptuel) les ressources mentales nécessaires à l'identification de leurs arguments comme des "systèmes intentionnels ». En ce sens, la théorie de l'esprit est sans aucun doute un candidat de choix. TagerFlusberg (1999) indique clairement que ces items lexicaux sont souvent absents du discours des enfants atteints d'autisme. S'il est acquis que la syntaxe, notamment les relations prédicat-argument, est «projetée à partir du lexique » (Chomsky, 1981, 1995), l'absence même de ce concept débouchera nécessairement sur une difficulté à décoder la syntaxe des verbes psychologiques (Belletti, A., Rizzi, L., 1988). Au vu de telles spéculations, il est fort à parier que le rôle de la théorie de l'esprit va bien au-delà du module inférentiel de Sperber et Wilson. La question se pose en outre de savoir comment ces mécanismes actuels pourraient raisonnablement refléter l'émergence du langage au niveau phylogénétique. Dans son essai Mildblindness (1996), Simon Baron-Cohen s'interroge brièvement sur ce que la théorie de l'esprit aurait pu jouer dans la genèse du langage articulé. Pour certains, cette faculté métareprésentationnelle a inévitablement été un élément déterminant dans l'évolution de la communication (Dubreuil, 2008). Outre le fait que la théorie de l'esprit assure la perception des intentions communicatives (pragmatique) de même que les états mentaux exprimés par les verbes psychologiques (syntaxe), c'est peut-être grâce à elle qu'un lien de cohésion sociale suffisamment solide se serait développé entre les individus pour permettre la transmission conceptuelle, symbolique, et - finalement - linguistique. A ce stade, seule une investigation minutieuse et concertée entre la linguistique, l'ethnologie, la psychologie cognitive et évolutionniste pourra nous fournir une réponse.

\section{Conclusion - Une approche évolutionniste}

Comme je l'ai mentionné à plusieurs reprises, la suggestion de recherche esquissée dans le présent travail a une portée bien plus large que la problématique d'acquisition du langage chez l'homme moderne. Elle fait en particulier écho aux propositions de Tomasello (2001), selon qui les capacités cognitives dont nous faisons usage aujourd'hui forment l'héritage naturel auquel notre phylogénie nous aurait prédisposés au cours de notre histoire. S'il en est ainsi, les connaissances acquises quant à notre faculté du langage résumeraient en quelque sorte les divers stades évolutifs par lesquels l'homme moderne serait passé depuis son apparition sur terre il y a de ça quelque 200000 ans. Si l'on accepte la description générativiste du langage comme résultante de notre biologie, il apparait tout naturel d'inclure celui-ci parmi les produits de notre évolution (Bickerton, 2005). La linguistique moderne n'a d'ailleurs pas l'apanage de ces positions. Les célèbres découvertes par les neurologues P. Broca (1861-1869) et C. Wernicke (1848-1905) de deux aires cérébrales potentiellement impliquées dans le traitement d'items linguistiques avaient déjà ouvert la voie vers une conception darwinienne du langage comme un instinct supérieur à ceux présents chez l'ensemble des espèces animales (Pinker, 1994). D'aucun peuvent même aisément entrevoir les premiers linéaments mentalistes du langage dans les travaux de Ferdinand de Saussure (1916), pour qui les signes linguistiques « [trouvent] leur siège dans le cerveau (p.32)». Du côté de l'infiniment petit, 
depuis la localisation du gène FOX P2 et les première tentatives d'y déceler une origine génétique au langage (Lai et al. 2000 ; Vargha-Khadem et al. 2005), plusieurs données particulièrement indicatives issues d'importantes recherches sur le génome humain ne cessent d'alimenter le débat quant à l'étendue du rôle joué par notre patrimoine génétique dans le développement de notre compétence cognitive et linguistique (Alarcon et al. 2008). A l'aune des mystères entourant encore la place du langage dans la nature humaine, une erreur de taille serait d'ignorer ces expériences, quand bien même celles-ci feraient en partie fausse route.

Le jeu en vaut la chandelle. Ce foisonnement d'informations nouvelles issu des efforts conjugués entre spécialistes du langage et de la cognition permettra non seulement de corriger le tir sur ce que nous considérons « inné » et « acquis ", mais aussi de multiplier les scénarios sur l'évolution du langage, parmi lesquels certains semblent gagner en autorité. Reprenons à ce titre le postulat nativiste de base de l'école chomskyenne en [1], et confrontons-le au ton plus nuancé du neuroconstructivisme, défendu par Steven Quartz et Terence Sejnowski (1997) et résumés dans Karmiloff-Smith (1998):

«For the staunch nativist, a set of genes specifically targets domain specific modules as the end product of their epigenesis (e.g. a syntactic module, a morphological module, or a more narrowly pre-specified module for, say, canonical linkage rules of grammar). Under this non-developmental view, the environment simply acts as a trigger for identifying and setting (environmentally-derived) native-tongue realizations of (pre-specified) parameters of universal grammar. The child is born expecting nouns, verbs, canonical linking rules, agreement between asymmetrical sentence elements, and so forth, but not yet knowing how they are realized in her/his native tongue. »

"The neuroconstructivist approach to normal and atypical development fully recognizes innate biological constraints but, unlike the staunch nativist, considers them to be initially less detailed and less domain-specific as far as higher levels of cognitive functions are concerned. Rather, development itself is seen as playing a crucial role in shaping phenotypical outcomes, with the protracted period of postnatal growth as essential in influencing the resulting domain specificity of the developing neocortex [...]. A different view is that although evolution has prespecified many constraints on development, it has made the human neocortex increasingly flexible and open to learning during postnatal development. In other words, evolution is argued to have selected for adaptive outcomes and a strong capacity to learn, rather than prior knowledge. Within such a perspective, it is more plausible to think in terms of a variety of what one might call domainrelevant mechanisms that might gradually become domain-specific as a result of processing different kinds of input. »

L'avantage de cette approche "hybride » est qu'il est maintenant aisé d'envisager le langage comme la parcelle d'un ensemble plus large de facultés mentales, dont le statut inné/acquis doit encore être (dans la mesure du possible) partagé et démontré. D'autre part, s'il nous est aujourd'hui donné de classer la théorie de l'esprit parmi les éléments fondamentaux de notre capacité linguistique au sens large, observer la façon dont ces facultés se développent ou manquent de se développer fournira certainement nombre d'indications quant au terrain cognitif propice sur lequel repose notre faculté du langage. Et le travail ne manque pas si l'on se sait prêt, par exemple, à « disséquer » la théorie de l'esprit en ses sous-composantes, telles la faculté d'abstraction, d'orientation ou de conscience sociale, sans parler des mécanismes à l'origine de cette si mystérieuse "récursivité ", que beaucoup considèrent comme noyau dur de notre grammaire mentale. La richesse et la diversité de ces réflexions, aussi nobles les unes que les autres, augure de débats prometteurs sur la nature et les arcanes de l'esprit humain. 


\section{BIBLIOGRAPHIE}

ALARCON et al., (2008), Linkage, Association, and Gene-Expression Analyses Identify CNTNAP2 as an Autism-Susceptibility Gene, in The American Journal of Human genetics 82, pp. 150-159.

ATKINSON, M., (1992), Children's Syntax: An Introduction to Principles and Parameters, chap. 4, Oxford, Blackwell.

AUROUX, S., DESCHAMPS, J., KOULOUGHLI, D., (2004), La Philosophie du langage, Paris, PUF.

BAKER, M., (1997), Thematic Roles and Syntactic Structure, In: Elements of Grammar (L.

Haegeman, éd.), Dordrecht, Kluwer.

BARON-COHEN, S., LESLIE, A. M., FRITH, U., (1985), Does the Autistic Child have a Theory of Mind?, in Cognition (1985) 37-46.

BALTAXE, C., (1977), Pragmatic Deficits in the Language of Autistic Adolescents, in Journal of Paediatric Psychology 2, pp. 176-180.

BALTAXE, C., (1984), Use of contrastive stress in normal, aphasic and autistic children, in Journal of Speech and Hearing Research 27, pp. 97-105.

BARON-COHEN, S., (1996), Mindblindness: An Essay on Autism and Theory of Mind, Cambridge, MIT Press.

BELLETTI, A., RIZZI, L., (1988), Psych-verbs and theta-theory, Natural Language and Linguistic Theory 6(3), pp. 291-352.

BICKERTON, D., (2007) Language evolution: A brief guide for linguists, in Lingua 117, pp. 510-526. BLOOM, P., (2000), How Children Learn the Meaning of Words, Cambridge, MIT Press.

BOTTING, N., CONTI-RAMSDEN, G., (1999), Pragmatic Language Impairment without Autism, in Autism 3, pp. 371-396.

CHOMSKY, N., (1957), Syntactic Structures, Berlin, Mouton.

CHOMSKY, N., (1964), Aspects of the Theory of Syntax, Cambridge, MIT Press.

CHOMSKY, N., (1969), Un Compte rendu du « Comportement verbal » de B.F. Skinner, in Langages 16 , pp. 16-49.

CHOMSKY, N., (1981), Lectures on Government and Binding, Dordrecht, Foris.

CHOMSKY, N., (1995), The Minimalist Program, Cambridge, MIT Press.

CHOMSKY, N., (1968), Language and Mind, Cambridge, MIT Press.

COETZEE, J. M., Souvenirs du Texas, in 2007, Doubler le cap, trad. franç. de J. L. Cornille, Paris, Édition du Seuil.

CASASANTO, D., (2005), Crying « Whorf », in Science (307), pp. 1721-1772.

COSMIDES, L., TOOBY, J., (1992), The Adapted Mind: Evolutionary Psychology and the Generation of Culture, Oxford, Oxford University Press.

CRAIN, S., (2006), Brigid's interview of Stephen Crain, entretien entre Brigid Mullane et Stephen Crain, document audio, [http://www.archive.org/details/stephen_crain]. 
CULLICOVER, P.W., JACKENDOFF, R., (2005), Simpler Syntax, Oxford, Oxford University Press.

De BOYSSON-BARDIES, B., (1996), Comment la parole vient aux enfants, Paris, Odile Jacob.

DEMUTH, K., (1987), Discourse functions of word order in Sesotho acquisition, in R. Tomlin (ed.), Coherence and Grounding in Discourse. Proceedings of a Symposium on Discourse, Cognition and Syntax, Eugene, Oregon. Amsterdam: John Benjamins. pp. 91-108.

DUBREUIL, B., (2008) What do modern behaviors in Homo Sapiens imply for the Evolution of Language?, In: The Evolution of Language: Proceedings of the 7Th International Conference, Barcelona, World Scientific.

EVERETT, D., L., (1986), Pirahã, in Desmond C. Derbyshire and Geoffrey K. Pullum (eds.), Handbook of Amazonian languages, vol. 1, 200-325. Berlin: Mouton de Gruyter.

FAY, W. H., (1979), Personal Pronouns and the Autistic Child, in Journal of Autism and Developmental Disorders $n^{\circ} 3$.

FINE, J., BARTOLUCCI, G., SZATMARI, P., GINSBERG, G., (1994), Cohesive Discourse in Pervasive Developmental Disorders, in Journal of Autism and Developmental Disorders 24, pp. 315-329.

FISETTE, D., POIRIER, P., (2000), Philosophie de l'esprit : État des lieux, Paris, Vrin.

FODOR, J., (1983), The Modularity of Mind: An Essay on Faculty Psychology, Cambridge, MIT Press.

GANGER, J.B., DUNN, S., GORDON, P., (2005/in press) Genes take over when the input fails: A twin study of the passive. Proceedings of the $28^{\text {th }}$ Annual Boston University Conference on Language Development, New York, Columbia University.

GORDON, P., (2004), Numerical Cognition without Words: Evidence from Amazonia, in Science Express pp. 1-10.

GORDON, P., (2005), Response to D. Casasanto's Crying “Whorf”, in Science (307), p. 1772.

GRICE, H. P., (1989), Studies in the Way of Words, Cambridge: Harvard University Press.

HALLIDAY, M. A. K., MATTHIESSEN C. M. I. M., (2004), An Introduction to Functional Grammar, 3d ed., Londres, Arnold.

HAUSER, M., CHOMSKY, N., FITCH, T. W., (2002) The Faculty of Language: What is it, Who has it, and How did it evolve?, in Science 298, pp. 1569-1579.

JACKENDOFF, R., An agenda for studying social cognition, Conférences: Mental Structures: Language, Society, Consciousness, Institut Jean Nicod, 31 mars 2003.

JACKENDOFF, R., Reintegrating Generative Grammar, Conférences: Mental Structures: Language, Society, Consciousness,Institut Jean Nicod, 27 mars 2003.

Jackendoff, R., (2007)., A parallel architecture perspective on language processing, in Brain Research n. 1146, pp. 2-22.

JAKOBSON, R., (1963), Essai de linguistique générale, Paris, Les Éditions de minuit.

KARMILOFF-SMITH, (1998), Development itself is the key to understanding developmental disorders, in Trends in Cognitive Sciences 2, pp. 389-398.

LAI, C., FISCHER, S., HURST, J., LEVY, E., HODGSON, S., FOX, M., JEREMIAH, S., POVEY, S., JAMISON, D., GREEN, E., VARGHA-KHADEM, F., MONACO, A., (2000), The SPCH1 region on human 7q31: genomic characterization of the critical interval and localization of translocations 
associated with speech and language disorder. In American Journal of Human Genetics 67 (2), pp. $357-68$.

LECRLERQ, B., L'Homme, un singe comme les autres ?, in Pour la science n57, Octobre-décembre 2007.

LENNEBERG, E., (1957), The Biological Foundations of Language, New York, John Wiley and Sons. LIGHTFOOT, D., (1987), The Language Lottery: Toward a Biology of Grammars, Cambridge, MIT Press.

LOCKE, J., ([1690] 1983), Essais sur l'entendement humain, trad. Franç. De P.Coste, Paris, Vrin. MERCIER, N., (2007), Je parle donc je suis, in Québec Science.

ÖZGEN, E., (2004), Language, Learning and Color Perception, in Current Directions in Psychological Science 13 (3), pp. 95-98.

PEPPE, S., McCANN, J., GIBBON, F., O'HARE, A., RUTHERFORD, M., (2006), Assessing prosodic and pragmatic ability in children with high-functioning autism, in Journal of Pragmatics 38, pp. 1776-1791.

PEZETSKY, D., (1982), Paths and Categories, Thèse de doctorat, MIT.

PIERCE, S., BARTOLUCCI, G., (1977), A Syntactic Investigation of Verbal Autistic, Mentally Retarded, and Normal Children, in Journal of Autism and Childhood Schizophrenia 7, pp. 121-134. PINKER, S., (1987), The Bootstrapping Problem in Language Acquisition, in B. Mc Whinney, Mechanisms of Language Acquisition, Hillsdale, New York, Erlbaum.

PINKER, S., (1994), The Language Instinct, New York, Harper Perennial.

PINKER, (1995), Language Acquisition, in Gleitman, Hila \& Lieberman, An Invitation to Cognitive Science 1, Language, Cambridge, MIT Press.

PINKER, S., JACKENDOFF, R., (2005), The Faculty of Language: what's special about it, in Cognition 96, pp. 201-236.

PINKER, S., ULLMAN, M., (2002), The Past and the Future of Past Tense, in Trends in Cognitive Sciences 6, pp. 456-463.

PREMACK, D. \& WOODRUFF, G. (1978). Does the chimpanzee have a theory of mind?, in Behavioral Brain Sciences, 1, pp. 515-526.

QUARTZ, S., SEJNOWSKI, T. J., (1997), The Neural Basis for Cognitive Development: A Constructivist Manifesto, in Brain and Behavioral Sciences, 20, pp. 537-596.

SAMPSON, G., (2005), The 'Language Instinct' Debate, Londres, Continuum.

SAXE, R., KANWISHER, N, (2003), People thinking about thinking people - The role of the temporo-parietal junction in "theory of mind", in. NeuroImage 19, pp. 1835-1842.

De SAUSSURE, F., (1913), Cours de linguistique générale, Paris, Payot.

SCHANK, R., ABELSON, R. B., (1977), Scripts, plans, goals and understanding: an inquiry into human knowledge structures, Hillsdale.

SCOTT, S., (accepted november 2001), Chimpanzee Theory of Mind: A Proposal from the Armchair, Ottawa, Carleton University.

SEARLE, J., R., (1972), Chomsky's Revolution in Linguistics, in The New York Review of Books. SPERBER, D., WILSON, D., (1981), La pertinence, Paris, Les Editions de Minuit. 
SPERBER, D., (oct. 2002) Anthropologie cognitive, langage et communication, évolution, entretien à la Maison des Sciences de L'Homme, Institut Jean Nicod.

SURIAN, L., BARON-COHEN, S., VAN DER LELY, H., (1996), Are Children with Autism Deaf to Gricean Maxims?, in Cognitive Neuropsychiatry 1, pp. 51-71.

TAGER-FLUSBERG, H., (1999), Language Development in Children with Autism, In: Methods for Studying Language Production, (L. Menn, Ratner, B. N. éds), New York, Lawrence Erlbaum Associates.

TATTERSALL, I., (1998), L'émergence de l'homme (trad. franç. Marcel Blanc), Paris, Gallimard.

TATTERSALL, I., Nombreux hier, seuls aujourd'hui, in Pour la science, $n^{\circ} 57$, Octobre/Décembre 2007.

TOMASELLO, M., (2002), The item-based nature of children's early language development, in Trends in Cognitive Sciences 4, pp. 156-163.

TELLIER, C., (2003), Éléments de syntaxe du français : Méthodes d'analyse en grammaire générative, $2^{\mathrm{e}}$ éd., Montréal, Gaëtan Morin Éditeur.

TOMASELLO, M., BROOK, P.J., (1999), Early syntactic development: A Construction Grammar approach. In M. Barrett (Ed.), The Development of Language (161-190). Philadelphia, PA: Psychology Press.

TOMASELLO, M., (2001), The Cultural Origins of Human Cognition, Cambridge, Harvard University Press.

VAN VALIN, R. D., (2005), Exploring the Syntax-Semantics Interface, Cambridge, Cambridge University Press.

VARGHA-KHADEM, F., GADIAN, D., COPP, A., MISHKIN, M., (2005), FOXP2 and the neuroanatomy of speech and language, in Nature Reviews Neuroscience 6, pp. 131-137.

WHORF, B. L., (1956), Linguistique et anthropologie, trad. franç. de C. Carme, Paris, Denoël.

WITTGENSTEIN, L., (1961), Investigations philosophiques, trad. P. Klossowski, Gallimard, Paris.

The Selected Writings of Edward Sapir, ed. D. G. Mandelbaum, Berkeley, University of California Press, 1951.

\section{NOTES}

1. An agenda for studying social cognition, Traduction choisie.

Je remercie tout particulièrement Daniel Valois, Phaedra Royle, Marie Labelle, Denis Bouchard, Michel Seymour, Claire Durand, ainsi que les deux relecteurs de la revue CORELA pour leurs précieuses suggestions apportées dans la rédaction du présent article.

2. Traduction choisie.

3. Communication personnelle.

4. J'emploie ici le terme "sémantique " dans un sens suffisamment large pour englober les aspects pragmatiques qui m'intéressent dans le présent texte.

5. Traduction choisie. 


\section{RÉSUMÉS}

Le présent article vise à proposer une synthèse critique du débat «nature vs nurture » sur la question du langage, de son acquisition et de sa place au sein de la nature et de la biologie humaine. L'accent y est mis sur une étude "mentaliste » du langage telle que proposée par l'école générativiste, sans pour autant en exclure le rôle du milieu social et contextuel dans le développement linguistique des individus. L'étude de ce dernier se justifie d'une part au travers des nombreuses découvertes relatives aux déficits pragmatiques et communicatifs des populations atteintes de troubles envahissants du développement ainsi que par les hypothèses proposées dans le cadre de l'évolution humaine en général. Une investigation approfondie de ces déficits alliée aux recherches actuelles sur la théorie de l'esprit et sur le siège du langage dans la cognition ne peut qu'augurer de débats riches et prometteurs sur les mystères entourant l'esprit humain.

The present article aims at providing a critical overview of the nature-nurture debate on language, language acquisition and its place within human nature and biology. This paper focuses on a mentalist approach to the study of language, without excluding the role of social and contextual environments in the individual's language acquisition process. This is justified, not only by the numerous discoveries made while studying pragmatic and communicative deficits in people with pervasive developmental disorders, but also by hypotheses put forth regarding human evolution in general. A thorough investigation of these disorders, Theory of Mind and general cognition will undoubtedly be the source of promising debates on the mysteries of the human mind.

\section{INDEX}

Mots-clés : acquisition du langage, grammaire universelle, théorie de l'esprit, syntaxe, sémantique, pragmatique, sciences cognitives

Keywords : language acquisition, universal grammar, theory of mind, syntax, semantics, pragmatics, cognitive science

\section{AUTEUR}

\section{NICOLAS BOURGUIGNON}

Université de Montréal, CHU Sainte-Justine (Montréal), Center for Research on Language Mind and Brain, (Université McGill) 